PROCEEDINGS OF THE

AMERICAN MATHEMATICAL SOCIETY

Volume 126, Number 12, December 1998, Pages 3507-3511

S 0002-9939(98)05010-2

\title{
EIGENVALUES OF A STURM-LIOUVILLE PROBLEM AND INEQUALITIES OF LYAPUNOV TYPE
}

\author{
CHUNG-WEI HA
}

(Communicated by Hal L. Smith)

\begin{abstract}
We consider the eigenvalue problem $u^{\prime \prime}+\lambda u+p(x) u=0$ in $(0, \pi)$, $u(0)=u(\pi)=0$, where $p \in L^{1}(0, \pi)$ keeps a fixed sign and $\|p\|_{L^{1}}>0$, and we obtain some lower and upper bounds for $\|p\|_{L^{1}}$ in terms of its nonnegative eigenvalues $\lambda$. Two typical results are: (1) $\|p\|_{L^{1}}>\sqrt{\lambda}|\sin \sqrt{\lambda} \pi|$ if $\lambda>1$ and is not the square of a positive integer; (2) $\|p\|_{L^{1}} \leq 16 / \pi$ if $\lambda=0$ is the smallest eigenvalue.
\end{abstract}

\section{INTRODUCTION}

We consider the eigenvalue problem

$$
u^{\prime \prime}+\lambda u+p(x) u=0 \quad \text { in }(0, \pi), \quad u(0)=u(\pi)=0,
$$

where $p \in L^{1}(0, \pi)$. It follows from the classical inequality of Lyapunov that if $\lambda=0$ is an eigenvalue of the problem (1), then necessarily

$$
\|p\|_{L^{1}}>\pi / 4 \text {. }
$$

Over the years there have appeared a number of improvements and extensions of this interesting result (see, e.g. [1], [2]). The purpose of this paper is to extend the original Lyapunov inequality in yet another direction. We treat the problem when $\lambda$ is any nonnegative eigenvalue of (1).

We assume throughout that

$$
p \in L^{1}(0, \pi),\|p\|_{L^{1}}>0 \text { and either } p \geq 0 \text { or } p \leq 0 \text { a.e. on }(0, \pi) .
$$

Our main results are given in $\S 2$ in which some lower bounds for $\|p\|_{L^{1}}$ are obtained in terms of the nonnegative eigenvalues of (1). In $\S 3$ we derive some upper bounds for $\|p\|_{L^{1}}$ which ensure an eigenvalue $\lambda \in[0,1]$ of (1) to be the smallest eigenvalue. For their proofs, we use basic properties of some boundary value problems and their Green functions (see, e.g. [7]). It was observed in [5] that (2) could be proved using the Green function method. We refer to [6, Chap. 2] for other interesting results relating to the eigenvalue problem (1). This work is motivated by a study on the solvability of some nonlinear boundary value problems (see [3], [4]).

Received by the editors September 23, 1996.

1991 Mathematics Subject Classification. Primary 34L15, 34L20.

(C) 1998 American Mathematical Society 


\section{INEQUALITIES OF LYAPUNOV TYPE}

In this section some inequalities of Lyapunov type are obtained. We always denote by $n$ a generic positive integer and consider the cases $\lambda \neq n^{2}$ and $\lambda=n^{2}$ separately in Theorems 1 and 2 below.

We suppose first that $\lambda \neq n^{2}$. We recall that for any $h \in L^{1}(0, \pi)$, the boundary value problem

$$
u^{\prime \prime}+\lambda u+h(x)=0 \quad \text { in }(0, \pi), \quad u(0)=u(\pi)=0
$$

has a unique solution $u$. More precisely, if $\lambda>0$, we have

$$
u(x)=\int_{0}^{\pi} G(x, \xi, \lambda) h(\xi) d \xi
$$

where

$$
G(x, \xi, \lambda)= \begin{cases}\frac{1}{\sqrt{\lambda} \sin \sqrt{\lambda} \pi} \sin \sqrt{\lambda} \xi \sin \sqrt{\lambda}(\pi-x) & \text { if } 0 \leq \xi \leq x \\ \frac{1}{\sqrt{\lambda} \sin \sqrt{\lambda} \pi} \sin \sqrt{\lambda} x \sin \sqrt{\lambda}(\pi-\xi) & \text { if } x \leq \xi \leq \pi\end{cases}
$$

is the Green function for the problem (4). It follows by a simple calculation that

$$
|G(x, \xi, \lambda)|< \begin{cases}\frac{1}{2 \sqrt{\lambda}} \tan \frac{\sqrt{\lambda}}{2} \pi & \text { if } 0<\lambda<1 \\ \frac{1}{\sqrt{\lambda}}|\csc \sqrt{\lambda} \pi| & \text { if } \lambda>1\end{cases}
$$

holds a.e. on the square $0 \leq \xi, x \leq \pi$.

Theorem 1. If $\lambda>0$ is an eigenvalue of (1), $\lambda \neq n^{2}$, then

$$
\|p\|_{L^{1}}> \begin{cases}2 \sqrt{\lambda} \cot \frac{\sqrt{\lambda}}{2} \pi & \text { if } 0<\lambda<1 \\ \sqrt{\lambda}|\sin \sqrt{\lambda} \pi| & \text { if } \lambda>1\end{cases}
$$

Proof. We assume that $p \geq 0$ a.e. on $(0, \pi)$. The other case can be proved similarly. Let $u$ be an eigenfunction of (1) corresponding to $\lambda$. Since $u$ is a nontrivial solution to (4) with $h(x)=p(x) u$, it follows from the remark above that

$$
u(x)=\int_{0}^{\pi} G(x, \xi, \lambda) p(\xi) u(\xi) d \xi .
$$

Taking the inner product in $L^{2}(0, \pi)$ of $(7)$ with $p(x) u$, we have

$$
\begin{aligned}
\int_{0}^{\pi} p(x)|u(x)|^{2} d x & =\int_{0}^{\pi} \int_{0}^{\pi} G(x, \xi, \lambda) p(\xi) u(\xi) p(x) u(x) d \xi d x \\
& <m(\lambda)^{-1}\left(\int_{0}^{\pi} p(x)|u(x)| d x\right)^{2}
\end{aligned}
$$

where $m(\lambda)$ is the function defined by the right-hand side of (6), which is reciprocal to that of the right-hand side of (5). Hence

$$
\begin{aligned}
\int_{0}^{\pi} p(x)|u(x)| d x & \leq\left(\int_{0}^{\pi} p(x)|u(x)|^{2} d x\right)^{\frac{1}{2}}\left(\int_{0}^{\pi} p(x) d x\right)^{\frac{1}{2}} \\
& <m(\lambda)^{-\frac{1}{2}}\left(\int_{0}^{\pi} p(x)|u(x)| d x\right)\left(\int_{0}^{\pi} p(x) d x\right)^{\frac{1}{2}} .
\end{aligned}
$$

By hypothesis $\int_{0}^{\pi} p(x)|u(x)| d x \neq 0$, and so the result follows. 
Obviously the inequality (2) follows from a similar line of arguments using instead the corresponding Green function

$$
G(x, \xi, 0)= \begin{cases}\frac{1}{\pi} \xi(\pi-x) & \text { if } 0 \leq \xi \leq x \\ \frac{1}{\pi} x(\pi-\xi) & \text { if } x \leq \xi \leq \pi\end{cases}
$$

As $G(x, \xi, \lambda)$ is continuous at $\lambda=0$, we have $\lim _{\lambda \rightarrow 0^{+}} m(\lambda)=4 / \pi$ as expected.

We now suppose that $\lambda=n^{2}$. We recall that for any $h \in L^{1}(0, \pi)$, the boundary value problem

$$
u^{\prime \prime}+n^{2} u+h(x)=0 \quad \text { in }(0, \pi), \quad u(0)=u(\pi)=0
$$

has a solution if and only if $\int_{0}^{\pi} h(x) \sin n x d x=0$. If this is the case, there exists a unique solution $u$ to $(9)$ such that $\int_{0}^{\pi} u(x) \sin n x d x=0$. More precisely, we have

$$
u(x)=\int_{0}^{\pi} G\left(x, \xi, n^{2}\right) h(\xi) d \xi
$$

where

$$
G\left(x, \xi, n^{2}\right)=-\xi \cos n \xi(\sin n x) / n \pi+ \begin{cases}\sin n \xi(\cos n x) / n & \text { if } 0 \leq \xi \leq x \\ \cos n \xi(\sin n x) / n & \text { if } x \leq \xi \leq \pi\end{cases}
$$

is the Green function for the problem (9).

Theorem 2. If $\lambda=n^{2}$ is an eigenvalue of (1), then

$$
\|p\|_{L^{1}}>2 n \text {. }
$$

Proof. We assume that $p \geq 0$ a.e. on $(0, \pi)$. The other case can be proved similarly. Let $u$ be an eigenvalue of (1) corresponding to $\lambda=n^{2}$. Since $u$ is a nontrivial solution to $(9)$ with $h(x)=p(x) u$, it follows from the remark above that

$$
u(x)=(2 / \pi)\left(\int_{0}^{\pi} u(\xi) \sin n \xi d \xi\right) \sin n x+\int_{0}^{\pi} G\left(x, \xi, n^{2}\right) p(\xi) u(\xi) d \xi
$$

Moreover,

$$
\int_{0}^{\pi} p(x) u(x) \sin n x d x=0
$$

and so taking the inner product in $L^{2}(0, \pi)$ of $(10)$ with $p(x) u$, we have

$$
\begin{aligned}
\int_{0}^{\pi} p(x)|u(x)|^{2} d x & =\int_{0}^{\pi} \int_{0}^{\pi} G\left(x, \xi, n^{2}\right) p(\xi) u(\xi) p(x) u(x) d \xi d x \\
=(1 / n) & {\left[\int_{0}^{\pi} \int_{0}^{x} \sin n \xi(\cos n x) p(\xi) u(\xi) p(x) u(x) d \xi d x\right.} \\
+ & \left.\int_{0}^{\pi} \int_{x}^{\pi} \cos n \xi(\sin n x) p(\xi) u(\xi) p(x) u(x) d \xi d x\right]
\end{aligned}
$$

It follows by (11) again that

$$
\begin{aligned}
& {\left[\int_{0}^{\pi} \int_{0}^{x}+\int_{0}^{\pi} \int_{x}^{\pi} \cos n \xi(\sin n x) p(\xi) u(\xi) p(x) u(x) d \xi d x\right] } \\
= & \int_{0}^{\pi} \int_{0}^{\pi} \cos n \xi(\sin n x) p(\xi) u(\xi) p(x) u(x) d \xi d x=0
\end{aligned}
$$


and hence

$$
\begin{aligned}
\int_{0}^{\pi} p(x)|u(x)|^{2} d x & =(1 / n) \int_{0}^{\pi} \int_{0}^{x} \sin n(\xi-x) p(\xi) u(\xi) p(x) u(x) d \xi d x \\
& <(1 / 2 n)\left(\int_{0}^{\pi} p(x)|u(x)| d x\right)^{2}
\end{aligned}
$$

We obtain as in (8) that

$$
\int_{0}^{\pi} p(x)|u(x)| d x<(1 / \sqrt{2 n})\left(\int_{0}^{\pi} p(x)|u(x)| d x\right)\left(\int_{0}^{\pi} p(x) d x\right)^{\frac{1}{2}} .
$$

By hypothesis $\int_{0}^{\pi} p(x)|u(x)| d x \neq 0$, and so the result follows.

We note that the assumption $\|p\|_{L^{1}}>0$ is essential for Theorem 2 to hold. Clearly $u=\sin n x$ is an eigenfunction of (1) corresponding to $n^{2}$ when $p=0$ a.e. on $(0, \pi)$.

\section{UPPER BOUNDS FOR $\|p\|_{L^{1}}$}

In this section we obtain some upper bounds of $\|p\|_{L^{1}}$ which are sufficient for an eigenvalue $\lambda \in[0,1]$ of (1) to be the smallest eigenvalue. We recall that if $\lambda$ is the smallest eigenvalue of (1), the eigenfunction $u$ corresponding to $\lambda$ has no zero in $(0, \pi)$. This is the property our proof is based on.

Theorem 3. Let $0 \leq \lambda \leq 1$. If $\lambda$ is an eigenvalue of (1) and

$$
\|p\|_{L^{1}} \leq \begin{cases}\frac{16}{\pi} & \text { if } \lambda=0 \\ 4 \sqrt{\lambda} \cot \frac{\sqrt{\lambda}}{4} \pi & \text { if } 0<\lambda \leq 1\end{cases}
$$

then $\lambda$ is the smallest eigenvalue.

Proof. Let $u$ be an eigenfunction of (1) corresponding to $\lambda$. We suppose on the contrary that $u$ has a zero $l \in(0, \pi)$ and define

$$
v(x)=u\left(\frac{l}{\pi} x\right), \quad w(x)=u\left(\frac{\pi-l}{\pi} x+l\right) .
$$

Then $v$ and $w$ are nontrivial solutions of (1) with $\lambda$ replaced by $\left(\frac{l}{\pi}\right)^{2} \lambda$ and $\left(\frac{\pi-l}{\pi}\right)^{2} \lambda$, $p(x)$ replaced by $\left(\frac{l}{\pi}\right)^{2} p\left(\frac{l}{\pi} x\right)$ and $\left(\frac{\pi-l}{\pi}\right)^{2} p\left(\frac{\pi-l}{\pi} x+l\right)$, respectively.

If $0<\lambda \leq 1$, we apply Theorem 1 to obtain

$$
\int_{0}^{l}|p(x)| d x>2 \sqrt{\lambda} \cot \frac{l}{2} \sqrt{\lambda} ; \quad \int_{l}^{\pi}|p(x)| d x>2 \sqrt{\lambda} \cot \frac{\pi-l}{2} \sqrt{\lambda} .
$$

It follows by a simple calculation that

$$
\begin{aligned}
\int_{0}^{\pi}|p(x)| d x & >2 \sqrt{\lambda}\left[\cot \frac{l}{2} \sqrt{\lambda}+\cot \frac{\pi-l}{2} \sqrt{\lambda}\right] \\
& \geq 4 \sqrt{\lambda} \cot \frac{\sqrt{\lambda}}{4} \pi
\end{aligned}
$$

which contradicts (12). 
If $\lambda=0$, we apply (2) to obtain

$$
\begin{aligned}
\int_{0}^{\pi}|p(x)| d x & =\left[\int_{0}^{l}+\int_{l}^{\pi}|p(x)| d x\right] \\
& >4\left(\frac{1}{l}+\frac{1}{\pi-l}\right) \geq \frac{16}{\pi}
\end{aligned}
$$

which again contradicts (12). This completes the proof of the theorem.

\section{REFERENCES}

1. L. Cesari, Asymptotic behavior and stability problems in ordinary differential equations, Springer Verlag, Berlin, 1971. MR 50:2582

2. A.M. Fink and D.F. St. Mary, On an inequality of Nehari, Proc. Amer. Math. Soc. 21 (1969), 640-642. MR 39:1737

3. C.W. Ha and C.C. Kuo, On the solvability of a two point boundary value problem at resonance, Topol. Methods Nonlinear Anal. 1 (1993), 295-302. MR 94g:34028

4. C.W. Ha and C.C. Kuo, On the solvability of a two point boundary value problem at resonance, II, Topol. Methods Nonlinear Anal. to appear.

5. Z. Nehari, On the zeros of solutions of second order linear differential equations, Amer. J. Math. 76 (1954), 689-697. MR 16:131f

6. J. Pöschel and E. Trubowitz, Inverse spectral theory, Academic Press, Orlando, FL, 1987. MR 89b:34061

7. I. Stakgold, Green's functions and boundary value problems, John Wiley and Sons, New York, 1979. MR 80k:35002

Department of Mathematics, National Tsing Hua University, Hsin Chu, Taiwan

E-mail address: cwha@math.nthu.edu.tw 